\title{
Smart Decode-and-Forward Relaying with Polar Codes
}

\author{
Dimitrios S. Karas, Student Member, IEEE, Koralia N. Pappi, Student Member, IEEE, \\ and George K. Karagiannidis, Senior Member, IEEE
}

\begin{abstract}
Polar codes have recently been proven to be capacity achieving for the physically degraded relay channel and relevant coding schemes have been proposed in the literature. In this paper, we deal with the design of polar codes in decode-andforward relaying and prove that the selective transmission of the relay to the destination, based on the decision of a detector of erroneous decoding - a method which we call "smart" relaying - can significantly improve the error probability performance. Furthermore, we propose a design for the aforementioned detector, that can be applied on the successive cancellation decoding of polar codes. Simulation results are presented to illustrate the efficiency of the proposed method.
\end{abstract}

Index Terms-Polar codes, decode-and-forward relaying, successive cancellation.

\section{INTRODUCTION}

C HANNEL polarization has been introduced in the pioneering work of Arikan [1] as a method to construct capacity achieving codes for any binary-input discrete memoryless channel (B-DMC). A recursive construction method and a decoder design for polar codes were also proposed in [1].

Various coding schemes for decode-and-forward (DF) relaying have been proposed in the literature [2]. For example, some of them have employed Turbo [3] [4] and Low Density Parity Check (LDPC) codes [5]. The use of polar codes for the relay channel has also been explored in the literature. In [6], it was proven that polar codes are applicable for the degraded wiretap and relay channels and it was shown that polar codes perform better than a corresponding LDPC code. In [7], the use of polar codes for degraded relay channels was examined and it was presented how polar codes can implement block Markov coding. In [8], the design of polar codes for compressand-forward $(\mathrm{CF})$ relaying was investigated for Wyner-Ziv coding. The method that motivated the present paper was proposed in the remarkable work [9], where polar codes are shown to be applicable for DF and CF relaying, and code construction methods are proposed for both cases. Other selective transmission schemes for DF relaying have been proposed, such as LDPC based [10] and LLR based [11].

In this letter, we consider a DF cooperative relaying system which uses polar codes. An erroneous decoding detector is used at the relay, that determines whether the relay will transmit information to the destination or remain silent. We call this technique as "smart" relaying, and we prove and

Manuscript received September 4, 2013. The associate editor coordinating the review of this letter and approving it for publication was M. Xiao.

The authors are with the Department of Electrical and Computer Engineering, Aristotle University of Thessaloniki, 54124 Thessaloniki, Greece (e-mail: \{dkaras, kpappi, geokarag\}@auth.gr).

Digital Object Identifier 10.1109/WCL.2013.111213.130639

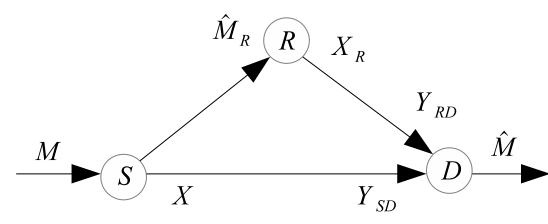

(a)

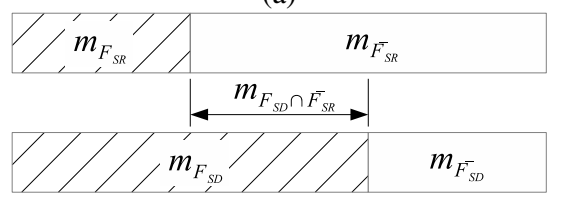

(b)

Fig. 1: Channel model and nested code structure.

verify through simulations that, if such a detector is used, then the error probability of the method proposed in [9] is an upper bound to the "smart" relaying one. Afterwards, we propose the design of such a detector, that can be used when a successive cancellation (SC) decoder for polar codes is considered [1].

\section{System AND Channel Model}

We consider a discrete memoryless relay channel with orthogonal receiver components, as depicted in Fig. 1a. It is consisted of a source $S$, a relay $R$ and a destination $D$. The message $M$, which is encoded to $X$ and transmitted by the source, is received by both relay and destination. The relay generates an estimation $\hat{M}_{R}$, re-encodes part of it to $X_{R}$ and transmits it to the destination. Finally, the destination produces its estimation of the original message, $\hat{M}$, by using the information received from both $S$ and $R$. Transmission occurs in two timeslots: $S$ transmits in the first timeslot and $R$ transmits in the second timeslot. The channels between $S$, $R$ and $D$ are denoted by $W_{S R}, W_{R D}$ and $W_{S D}$.

Next, we use the nested structure of polar codes proposed in [9] for DF relaying. An overview of this structure is presented in Fig. 1b, where the length of the code block is denoted by $N$. When encoding the information at the source, we select a frozen set of bits $F_{S R}$, where $\left|F_{S R}\right|<N$, whose indices are determined as

$$
F_{S R}=\left\{i \in\{0,1, \cdots, N-1\}: Z\left(W_{S R}^{(i)}\right) \geq \delta_{N}\right\},
$$

where $W_{S R}^{(i)}$ refers to the i-th channel constructed using channel combining and splitting, as described in Arikan's work [1]. $Z\left(W_{S R}^{(i)}\right)$ denotes the Bhattacharyya parameter of the $i$-th channel and $\delta_{N}=\frac{1}{N} 2^{-N^{\beta}}$, where $\beta$ is a constant such that $\beta<\frac{1}{2}$, so that the code is capacity achieving for the $W_{S R}$ channel. Conversely, $\bar{F}_{S R}$ denotes the set of information bits. 
We also select a set of indices so that the code would be capacity achieving for the $W_{S D}$ channel

$$
F_{S D}=\left\{i \in\{0,1, \cdots, N-1\}: Z\left(W_{S D}^{(i)}\right) \geq \delta_{N}\right\}
$$

and $\bar{F}_{S D}$ denotes the corresponding set of information bits. It should also be noted that if $W_{S D}$ is stochastically degraded compared to $W_{S R}$, then $F_{S R} \subseteq F_{S D}$ [12].

The coding scheme is performed as follows:

1) The source encodes the entirety of the information, denoted by $m_{\bar{F}_{S R}}$ in Fig. 1b, and transmits the encoded information to the relay and the destination.

2) The relay decodes the message and re-encodes the information, denoted by $m_{F_{S D} \cap \bar{F}_{S R}}$, by using a capacityachieving code for $W_{R D}$.

3) The relay transmits the re-encoded information to the destination, where it is decoded first and

4) The rest of the information received from the source is decoded, using the information received from the relay as frozen bits.

\section{ERroneous Decoding Detection for SC}

In this section, we propose a design of polar codes for "smart" DF relaying systems, when SC decoding is used. The difference of "smart" relaying with the above coding scheme is that the relay possesses a detector of erroneous decoding. Therefore in step 3, if erroneous decoding is detected, $R$ does not re-transmit and the entirety of the information is decoded from what is received only by $S$, as if it is a direct transmission without the presence of a relay. Note that, based on the coding scheme described in Section II, this is feasible for $D$. In the following, we use the same notations as in [1] to describe SC decoding. For example, $W_{N}^{(i)}$ denotes the $i$-th subchannel that is created by synthesizing $N$ copies of the channel $W$. The decoder has the received vector $y_{1}^{N}$, knows the frozen vector $u_{A^{c}}$ and generates an estimate $\hat{u}_{A}$ of the information vector $u_{A}$, where $A$ denotes the set of indices of information bits. Specifically, in each iteration the decoder applies the iterative channel transformation equations, [1, (22), (23)], to find the corresponding transition probabilities for each of the sub-channels formed by the channel synthesizing process. Afterwards, an estimate of the source element $u_{i}$ is generated by calculating the likelihood ratio

$$
L_{N}^{(i)}\left(y_{1}^{N}, \hat{u}_{1}^{i-1}\right)=\log \left[\frac{W_{N}^{i}\left(y_{1}^{N}, \hat{u}_{1}^{i-1} \mid 0\right)}{W_{N}^{i}\left(y_{1}^{N}, \hat{u}_{1}^{i-1} \mid 1\right)}\right] .
$$

Finally, the decision for the value of $u_{i}$ is made as follows

$$
\hat{u}_{i}=\left\{\begin{array}{cc}
0, & \text { if } L_{N}^{(i)}\left(y_{1}^{N}, \hat{u}_{1}^{i-1}\right) \geq 0 \\
1, & \text { otherwise. }
\end{array}\right.
$$

The detection method proposed in this paper sets a threshold for these likelihood ratios, defined by a parameter $s$ and discards the decoding result if a decision is made by using a likelihood ratio whose value exceeds this threshold. Specifically, we set a flag $F$ as

$$
F=\left\{\begin{array}{cc}
0, & \text { if }-s \leq L_{N}^{(i)}\left(y_{1}^{N}, \hat{u}_{1}^{i-1}\right) \leq s \\
1, & \text { otherwise. }
\end{array}\right.
$$

If $F=0$, then the decoding result is discarded and the relay does not transmit to the destination. The efficiency of this method is shown in Section V. To select the parameter $s$, we have to consider that lower values of $s$ will lead to a less selective detector that will only block transmissions where a LLR close to 0 was found and are therefore suitable for channels with high SNR, while higher values will be more selective and block more transmissions. It should be also noted that the proposed 'smart' relaying technique doesn't introduce any additional computational complexity at the relay, since it only relies on checking the LLR values generated by the decoder at the relay, which would be calculated whether we applied 'smart' relaying or not.

\section{SMART RELAYing}

Assume a relay that uses a method to detect whether its decoding is erroneous or not. Also, the probabilistic event of correct detection is denoted by $C$, and the corresponding probability is $P(C)$. Conversely, the complementary event $\bar{C}$ represents the event of incorrect detection, meaning that the relay detects that the decoding was correct, while it was erroneous, and vice versa. If the detector detects that a correct decoding has occurred, it transmits to the destination. Otherwise it remains idle during the second timeslot. We assume two cases for the relay:

Case A: There is no detector and the relay always transmits to the destination, as in [9]. Let $\mathcal{E}_{S R}$ and $\mathcal{E}_{R D}$ denote the events of erroneous estimation of the transmitted information between $S-R$ and $R-D$, respectively, and $\overline{\mathcal{E}}_{S R}$ and $\overline{\mathcal{E}}_{R D}$ denote their respective complementary events. Also, $\mathcal{E}_{A}$ is the event of erroneous estimation of the original message by the destination $D$ based on the combined information from $S$ and $R$.

Case B: An erroneous decoding detector with probability of success equal to $P(C)$ is present. Let $\mathcal{E}_{B}$ denote the event of erroneous estimation of the original message by the destination $D$.

Proposition 1: Assume the cases A and B described above, with error probabilities $P\left(\mathcal{E}_{A}\right)$ and $P\left(\mathcal{E}_{B}\right)$, respectively. If

$$
\zeta_{1} P\left(C, \mathcal{E}_{S R}\right) \geq \zeta_{2} P\left(\bar{C}, \overline{\mathcal{E}}_{S R}\right)
$$

where

$$
\zeta_{1}=P\left(\mathcal{E}_{A} \mid \mathcal{E}_{S R}\right)-P\left(\mathcal{E}_{B} \mid C, \mathcal{E}_{S R}\right)
$$

and

$$
\zeta_{2}=P\left(\mathcal{E}_{B} \mid \bar{C}, \overline{\mathcal{E}}_{S R}\right)-P\left(\mathcal{E}_{A} \mid \overline{\mathcal{E}}_{S R}\right)
$$

then

$$
P\left(\mathcal{E}_{B}\right) \leq P\left(\mathcal{E}_{A}\right) .
$$

Proof: In case B, we have to take the presence of the detector into consideration in order to calculate $P\left(\mathcal{E}_{B}\right)$ as

$$
P\left(\mathcal{E}_{B}\right)=P\left(\mathcal{E}_{B} \mid C\right) P(C)+P\left(\mathcal{E}_{B} \mid \bar{C}\right) P(\bar{C}) .
$$

However, $P(C)$ and the probability of the complementary event $P(\bar{C})$ are dependent on the transmission state between 
$S$ and $R$, so it holds that

$$
\begin{aligned}
P\left(\mathcal{E}_{B} \mid C\right) P(C) & =P\left(\mathcal{E}_{B} \mid C, \mathcal{E}_{S R}\right) P\left(\mathcal{E}_{S R} \mid C\right) P(C) \\
& +P\left(\mathcal{E}_{B} \mid C, \overline{\mathcal{E}}_{S R}\right) P\left(\overline{\mathcal{E}}_{S R} \mid C\right) P(C) \\
& =P\left(\mathcal{E}_{B} \mid C, \mathcal{E}_{S R}\right) P\left(C \mid \mathcal{E}_{S R}\right) P\left(\mathcal{E}_{S R}\right) \\
& +P\left(\mathcal{E}_{B} \mid C, \overline{\mathcal{E}}_{S R}\right) P\left(C \mid \overline{\mathcal{E}}_{S R}\right) P\left(\overline{\mathcal{E}}_{S R}\right)
\end{aligned}
$$

and similarly

$$
\begin{aligned}
P\left(\mathcal{E}_{B} \mid \bar{C}\right) P(\bar{C}) & =P\left(\mathcal{E}_{B} \mid \bar{C}, \mathcal{E}_{S R}\right) P\left(\bar{C} \mid \mathcal{E}_{S R}\right) P\left(\mathcal{E}_{S R}\right) \\
& +P\left(\mathcal{E}_{B} \mid \bar{C}, \overline{\mathcal{E}}_{S R}\right) P\left(\bar{C} \mid \overline{\mathcal{E}}_{S R}\right) P\left(\overline{\mathcal{E}}_{S R}\right)
\end{aligned}
$$

In (11), $P\left(\mathcal{E}_{B} \mid C, \overline{\mathcal{E}}_{S R}\right)$ denotes the probability of the event of erroneous detection at the destination in case $\mathrm{B}$, when the relay transmits a correctly received message. This event is identical to the event $\mathcal{E}_{A} \mid \overline{\mathcal{E}}_{S R}$ in case A, since the relay in case $\mathrm{A}$ always transmits. Thus it is

$$
P\left(\mathcal{E}_{B} \mid C, \overline{\mathcal{E}}_{S R}\right)=P\left(\mathcal{E}_{A} \mid \overline{\mathcal{E}}_{S R}\right),
$$

Similarly in (12), $P\left(\mathcal{E}^{\prime} \mid \bar{C}, \mathcal{E}_{S R}\right)$ denotes the probability the event of erroneous detection at the destination in case $\mathrm{B}$, when the relay transmits an erroneously received message. This event is identical to the event $\mathcal{E}_{A} \mid \mathcal{E}_{S R}$ in case A. Thus it is

$$
P\left(\mathcal{E}_{B} \mid \bar{C}, \mathcal{E}_{S R}\right)=P\left(\mathcal{E}_{A} \mid \mathcal{E}_{S R}\right),
$$

Combining (10), (11) and (12) and using (7), (8), (13) and (14) yields

$$
\begin{aligned}
P\left(\mathcal{E}_{B}\right) & =\left(P\left(\mathcal{E}_{A} \mid \mathcal{E}_{S R}\right)-\zeta_{1}\right) P\left(C \mid \mathcal{E}_{S R}\right) P\left(\mathcal{E}_{S R}\right) \\
& +P\left(\mathcal{E}_{A} \mid \overline{\mathcal{E}}_{S R}\right) P\left(C \mid \overline{\mathcal{E}}_{S R}\right) P\left(\overline{\mathcal{E}}_{S R}\right) \\
& +P\left(\mathcal{E}_{A} \mid \mathcal{E}_{S R}\right) P\left(\bar{C} \mid \mathcal{E}_{S R}\right) P\left(\mathcal{E}_{S R}\right) \\
& +\left(P\left(\mathcal{E}_{A} \mid \overline{\mathcal{E}}_{S R}\right)+\zeta_{2}\right) P\left(\bar{C} \mid \overline{\mathcal{E}}_{S R}\right) P\left(\overline{\mathcal{E}}_{S R}\right)
\end{aligned}
$$

Taking into consideration that

$$
\begin{aligned}
& P\left(C \mid \mathcal{E}_{S R}\right)+P\left(\bar{C} \mid \mathcal{E}_{S R}\right)=1 \\
& P\left(C \mid \overline{\mathcal{E}}_{S R}\right)+P\left(\bar{C} \mid \overline{\mathcal{E}}_{S R}\right)=1
\end{aligned}
$$

after some mathematical manipulations, (15) is reduced to

$$
\begin{aligned}
P\left(\mathcal{E}_{B}\right) & =P\left(\mathcal{E}_{A}\right)-\zeta_{1} P\left(C \mid \mathcal{E}_{S R}\right) P\left(\mathcal{E}_{S R}\right) \\
& +\zeta_{2} P\left(\bar{C} \mid \overline{\mathcal{E}}_{S R}\right) P\left(\overline{\mathcal{E}}_{S R}\right) .
\end{aligned}
$$

If (6) is valid, then (9) is derived from (18) and this concludes the proof.

\section{A. The Parameters $\zeta_{1}$ and $\zeta_{2}$}

Next, we make some observations on the parameters $\zeta_{1}$ and $\zeta_{2}$, which depend on the error probabilities of the $S-R, R-D$ and $S-D$ channels and the polar codes used in the system. In order to estimate $\zeta_{1}$ and $\zeta_{2}$ in a practical system, we can use information from channel estimation to perform simulations of the relay channel for both cases A and B. Specifically, for $\zeta_{1}$ holds

$$
\begin{aligned}
\zeta_{1} & =P\left(\mathcal{E}_{A} \mid \mathcal{E}_{S R}\right)-P\left(\mathcal{E}_{B} \mid C, \mathcal{E}_{S R}\right) \\
& =P\left(\mathcal{E}_{A} \mid \mathcal{E}_{S R}\right)-P\left(\mathcal{E}_{S D}\right)
\end{aligned}
$$

since in case $\mathrm{B}$, when the detector of the relay detects an erroneous transmission over $S-R$, the relay does not transmit.
Thus, the transmission is performed only over the channel $S-D$ and not the channel $R-D$, which corresponds to the direct communication case, without the presence of a relay. Consequently, the probability of erroneous detection corresponds to the probability of erroneous transmission over $S-D$.

Similarly, for $\zeta_{2}$ holds that

$$
\begin{aligned}
\zeta_{2} & =P\left(\mathcal{E}_{B} \mid \bar{C}, \overline{\mathcal{E}}_{S R}\right)-P\left(\mathcal{E}_{A} \mid \overline{\mathcal{E}}_{S R}\right) \\
& =P\left(\mathcal{E}_{S D}\right)-P\left(\mathcal{E}_{A} \mid \overline{\mathcal{E}}_{S R}\right)
\end{aligned}
$$

since in case $\mathrm{B}$, when the detector of the relay erroneously detects an erroneous transmission over $S-R$, the relay does not transmit. Thus again, $P\left(\mathcal{E}_{B} \mid \bar{C}, \overline{\mathcal{E}}_{S R}\right)=P\left(\mathcal{E}_{S D}\right)$.

From (19) and (20) we can make the following observations. First, in (19), $P\left(\mathcal{E}_{A} \mid \mathcal{E}_{S R}\right)$ is usually very high, since it refers to case $\mathrm{A}$, when the relay's information is wrong. The relay will transmit to the destination, and the incorrect information received from the relay is used as frozen. However, the probability that the information transmitted by the relay will be changed by the channel $R-D$ back to the original correct message is usually very low. This is evident since it is a prerequisite that the $R-D$ channel imposes such an error that the exact same bits of the codeword which were erroneously detected in the $S-R$ transmission, are now changed back to their original values.

In (20), $P\left(\mathcal{E}_{A} \mid \overline{\mathcal{E}}_{S R}\right)$ corresponds to the erroneous detection of the information by the destination in case A, when the relay retransmits correct information. For a degraded $S-D$ channel with respect to the $R-D$ channel and appropriate code design, compared to the direct communication between $S$ and $D$, we expect that the correct transmission from the relay will be beneficial for the system, and thus $P\left(\mathcal{E}_{A} \mid \overline{\mathcal{E}}_{S R}\right)<P\left(\mathcal{E}_{S D}\right)$. In this case, $P\left(\mathcal{E}_{S D}\right)>\zeta_{2}>0$. If $P\left(\mathcal{E}_{A} \mid \overline{\mathcal{E}}_{S R}\right)>P\left(\mathcal{E}_{S D}\right)$, then $\zeta_{2}<0$. Consequently, the sign of $\zeta_{2}$ is an indicator for whether the addition of a relay in case $\mathrm{A}$ is beneficial for the transmission or not, supposing that information is transmitted correctly over $S-R$.

The above observations lead to the following proposition: Proposition 2: If

$$
P\left(\mathcal{E}_{A} \mid \mathcal{E}_{S R}\right)>(k+1) P\left(\mathcal{E}_{S D}\right)
$$

where

$$
k=\frac{P\left(\bar{C}, \overline{\mathcal{E}}_{S R}\right)}{P\left(C, \mathcal{E}_{S R}\right)}
$$

then (6) holds.

Proof: In the case of (21), it is

$$
\zeta_{1}=P\left(\mathcal{E}_{A} \mid \mathcal{E}_{S R}\right)-P\left(\mathcal{E}_{S D}\right)>k P\left(\mathcal{E}_{S D}\right) .
$$

For $\zeta_{2}$, if $P\left(\mathcal{E}_{A} \mid \overline{\mathcal{E}}_{S R}\right)<P\left(\mathcal{E}_{S D}\right)$, then $P\left(\mathcal{E}_{S D}\right)>\zeta_{2}>0$, as discussed above. Also, if $P\left(\mathcal{E} \mid \overline{\mathcal{E}}_{S R}\right)>P\left(\mathcal{E}_{S D}\right)$, then $\zeta_{2}<0$. So when (23) holds, it is

$$
\zeta_{1}>k \zeta_{2} .
$$

Multiplying (24) by $P\left(C, \mathcal{E}_{S R}\right)$ yields (6) and this concludes the proof. 


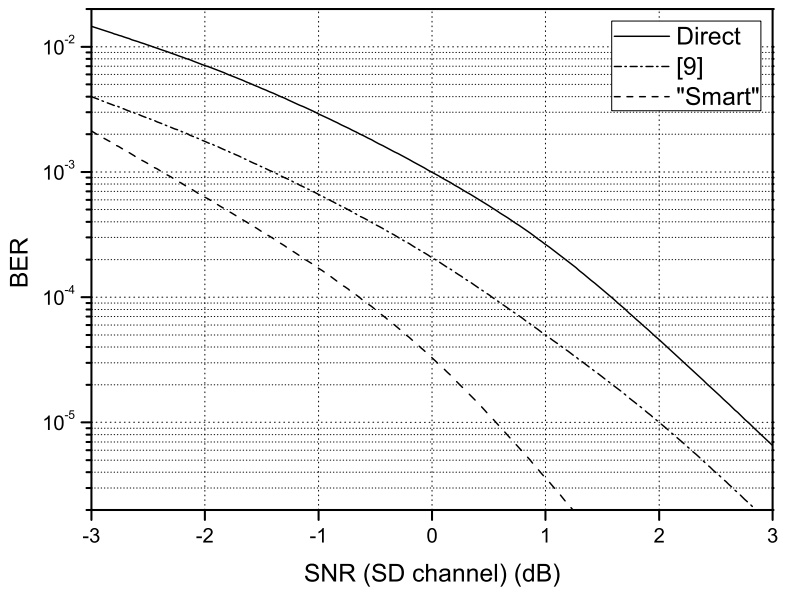

Fig. 2: Bit Error Rate for N=32 for "smart" relaying compared to [9] and to direct communication.

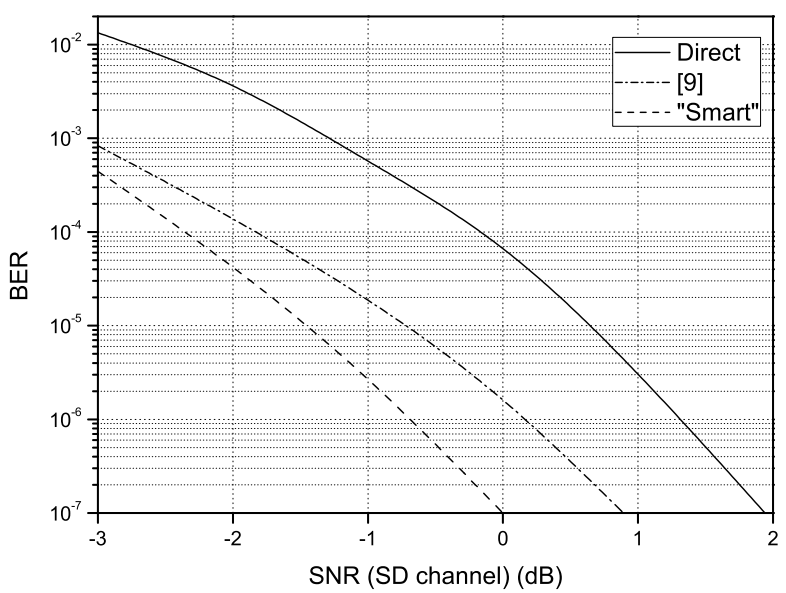

Fig. 3: Bit Error Rate for $\mathrm{N}=128$ for "smart" relaying compared to [9] and to direct communication.

\section{Simulation Results}

In this section, we present simulation results for the proposed "smart" relaying technique. We assume communication over an Additive White Gaussian Noise (AWGN) channel with hard-decision decoding at the destination. The $W_{S D}$ channel is physically degraded by $2 \mathrm{~dB}$ compared to the $W_{S R}$ and $W_{R D}$ channels. The cut-off parameter for the proposed method was set equal to $s=0.04$. Polar codes with length $N=32$ and $N=128$ were used and the destination is equipped with a SC decoder. The code rates that were used were $R_{S R}=0.25$ for source-relay transmission and $R_{R D}=0.125$ for relaydestination transmission. Results were also compared to those obtained when the method in [9] is used, and to those obtained when direct communication with no relay occurs. It should be noted that the 'Direct' curve has been included as an upper bound to the error probability for both methods.

We observe that the use of "smart" relaying leads to better error performance, compared to [9], while both methods provide improvement compared to the case where no relay was used. It was also confirmed through the simulations that, in every case, the proposed method for "smart" relaying satisfies the inequality (6) of Proposition 1, and inequality (21) of Proposition 2. In order to support this, some indicative
TABLE I: Simulation Results supporting Propositions 1 and 2

\begin{tabular}{|c||c|c|}
\hline$N=32$ & $S N R_{S D}=-3 \mathrm{~dB}$ & $S N R_{S D}=0 \mathrm{~dB}$ \\
\hline \hline$P\left(\mathcal{E}_{A} \mid \mathcal{E}_{S R}\right)$ & 0.9979 & 1 \\
\hline$P\left(\mathcal{E}_{S D}\right)$ & 0.157 & 0.00444 \\
\hline$k$ & 0.8187 & 1.831 \\
\hline$\zeta_{1}$ & 0.8409 & 0.99556 \\
\hline$\zeta_{2}$ & 0.1455 & 0.00432 \\
\hline$P\left(\mathcal{E}_{A}\right)$ & 0.04523 & 0.003 \\
\hline$P\left(\mathcal{E}_{B}\right)$ & 0.02479 & 0.00056 \\
\hline
\end{tabular}

simulation results are provided in Table I, averaged over 50,000 samples, which can easily verify both inequalities. Note that, even for low SNR values, $P\left(\mathcal{E}_{A} \mid \mathcal{E}_{S R}\right)$ is close to 1 , and $\zeta_{2}$ is positive, as discussed in Section IV-A.

\section{CONCLUSIONS}

We proposed a "smart" relaying technique with polar codes that involves an erroneous decoding detector at the relay and a detector construction for polar codes. It was proven that the selective transmission of the relay to the destination, based on the decision of the detector, can improve the error performance. Although "smart" relaying was designed to be used with a successive cancellation decoder, it can be easily extended to other decoding algorithms, that are based on the SC decoder, such as the one proposed in [13].

\section{REFERENCES}

[1] E. Arikan, "Channel polarization: a method for constructing capacityachieving codes for symmetric binary-input memoryless channels," IEEE Trans. Inf. Theory, vol. 55, no. 7, pp. 3051-3073, 2009.

[2] Y. Li, "Distributed coding for cooperative wireless networks: an overview and recent advances," IEEE Commun. Mag., vol. 47, no. 8, pp. 71-77, 2009.

[3] M. Valenti and B. Zhao, "Distributed turbo codes: towards the capacity of the relay channel," in Proc. 2003 IEEE Vehicular Technology Conference, pp. 322-326.

[4] M. Souryal and B. Vojcic, "Performance of amplify-and-forward and decode-and-forward relaying in Rayleigh fading with turbo codes," in Proc. 2006 IEEE International Conference on Acoustics, Speech and Signal Processing.

[5] P. Razaghi and W. Yu, "Bilayer low-density parity-check codes for decode-and-forward in relay channels," IEEE Trans. Inf. Theory, vol. 53, no. 10, pp. 3723-3739, Oct. 2007.

[6] M. Andersson, V. Rathi, R. Thobaben, J. Kliewer, and M. Skoglund, "Nested polar codes for wiretap and relay channels," IEEE Commun. Lett., vol. 14, no. 8, pp. 752-754, 2010.

[7] M. Karzand, "Polar codes for degraded relay channels," in Proc. 2012 International Zurich Seminar on Communications, p. 59.

[8] R. Blasco-Serrano, R. Thobaben, V. Rathi, and M. Skoglund, "Polar codes for compress-and-forward in binary relay channels," in Proc. 2010 IEEE Asilomar Conference on Signals, Systems and Computers, pp. 1743-1747.

[9] R. Blasco-Serrano, R. Thobaben, M. Andersson, V. Rathi, and M. Skoglund, "Polar codes for cooperative relaying," IEEE Trans. Commun., vol. 60, no. 11, pp. 3263-3273, 2012.

[10] Z. Chen and J. Yuan, "Selective cooperative schemes using quasicyclic LDPC codes over Rayleigh fading channels," in Proc. 2009 International Conference on Communication Software and Networks, pp. 654-658.

[11] R. Palat, A. Annamalai, and J. Reed, "Log-likelihood-ratio based selective decode and forward cooperative communication," in Proc. 2008 IEEE Vehicular Technology Conference, pp. 615-618.

[12] S. Korada and R. Urbanke, "Polar codes are optimal for lossy source coding," IEEE Trans. Inf. Theory, vol. 56, no. 4, pp. 1751-1768, 2010.

[13] I. Tal and A. Vardy, "List decoding of polar codes," in Proc. 2011 IEEE International Symposium on Information Theory, pp. 1-5. 